\title{
Monitoring the Development of HIFU-Induced Cavitation Activity
}

\author{
Caleb H. Farny ${ }^{*}$, R. Glynn Holt, and Ronald A. Roy \\ Department of Aerospace and Mechanical Engineering, Boston University, Boston MA. 02215
}

\begin{abstract}
One of the limitations of HIFU treatment for tissue necrosis is the difficulty in achieving a predictable lesion shape and size in a short amount of time. Simply increasing the HIFU intensity cannot solve this problem, as it leads to the formation of a so-called "tadpole"shaped lesion. Bubble shielding of the incident HIFU is one of the mechanisms implicated in the development of these deformed lesions; at super-nucleation pressures inertial cavitation will commence and the scattering from bubbles in the path of the ultrasound propagation will reflect the HIFU energy back towards the transducer. In the past we have employed a single focused, passive broadband transducer (PCD) to detect inertial cavitation activity at the HIFU focus in agar-graphite tissue phantoms. At sufficiently-high pressures, the inertial cavitation signal decreases over time, giving rise to the notion that bubble shielding is at fault for the signal decrease. Here we present evidence that bubble shielding is not the only mechanism behind such a change in the signal. As the medium heats up, inertial bubble collapses are cushioned by vapor and a decrease in signal amplitude from cavitation is to be expected. In order to evaluate the relative effects of the temperature rise and bubble shielding on bubble activity, we positioned a second PCD to sense noise emissions from various locations in the pre-focal region along the HIFU axis. A decline in the cavitation signal from the focus was accompanied by an increase in pre-focal bubble activity. The timescale for these changes suggests that both temperature and bubble shielding effects play a role in the bubble activity at the focus, and may provide information on how best to monitor the cavitation signal and ultimately provide feedback information necessary to control the HIFU insonation parameters to avoid bubble shielding.
\end{abstract}

Keywords: HIFU, bubbles, cavitation, hyperthermia, heating

PACS: 47.55.BX, 43.80.GX, 43.80.SH

\section{INTRODUCTION}

High-intensity focused ultrasound (HIFU) shows promise as a noninvasive therapy for a number of medical conditions. One of the chief problems with using HIFU for tumor necrosis is the difficulty in predicting the lesion shape. While lesions formed by pure absorptive heating from a HIFU source should possess a long, narrow cigarshaped pattern, in vivo and in vitro HIFU treatments often produce lesions shaped more like tadpoles-narrow in the focus and increasingly wide and bulbous closer to the transducer [1]. A leading explanation for this type of deformation suggests that bubbles nucleated by the HIFU act to both scatter the incoming pressure waves and effectively change the medium such that an impedance mismatch at the proximal edge

"email address: cfarny@bu.edu

CP829, Therapeutic Ultrasound: $5^{\text {th }}$ International Symposium on Therapeutic Ultrasound edited by G. T. Clement, N. J. McDannold, and K. Hynynen

(C) 2006 American Institute of Physics 0-7354-0321-X/06/\$23.00 
of the bubble cloud causes boundary reflections, shielding the focus from acoustic exposure and increasing the pressure pre-focally [1,2].

Experimental and theoretical investigations into the role of inertially-collapsing bubbles have shown that bubbles can locally enhance the heating [3,4], which suggests that if properly positioned [5], bubbles can assist HIFU treatment. Indeed, the high intensities commonly employed in HIFU therapy can make it difficult to avoid the onset of inertial cavitation. In order to better understand how bubbles can impact HIFU treatment, it is useful to monitor the cavitation bubble field and understand which factors affect the bubble dynamics. Our previous work in this area [6] employed a single passive cavitation detector (PCD), which is a focused broadband transducer used to detect emissions from inertial bubble collapses. Here we report on efforts to model the effect of temperature on the bubble dynamics and on the implementation of a second PCD to monitor the pre-focal bubble activity along the HIFU axis.

\section{THEORY}

Over time, the amplitude of inertial cavitation activity in the HIFU focus tends to diminish as the tissue heats up. We initially hypothesized that this was due to bubbles forming along the acoustic path, shielding the HIFU focus. This view is bolstered by the fact that the probability of nucleating cavitation increases as temperature rises, for the tissue outgasses. However, hot bubbles also contain elevated vapor pressures, which serve to cushion otherwise inertial collapses. The net result is less broadband noise and reduced bubble-enhanced heating. It is important, therefore, to investigate the role of temperature in HIFU-driven bubble dynamics.

We sought to estimate the radiated acoustic power from inertial cavitation as a function of ambient temperature. Employing a model describing the nonlinear bubble dynamics [7,8], we considered a small (sub-resonant size) air bubble in water insonated at a frequency and pressure amplitude of $1 \mathrm{MHz}$ and $3 \mathrm{MPa}$ respectively. The temperature dependencies of sound speed [9], vapor pressure [10], density [10], thermal conductivity [11], viscosity [12] and surface tension [13] were included in the calculations. Empirical fits of the temperature dependence were used when available, and look-up tables were used otherwise. The model was strictly valid up to $50^{\circ} \mathrm{C}$, for the effects of vapor condensation and evaporation were not accounted for. We evaluated the temperature-dependent bubble dynamics in steps of $10^{\circ} \mathrm{C}$ from $20-100^{\circ}$ C. Since evaporation and condensation was neglected, the higher temperature results should be regarded as ballpark estimates only.

\section{EXPERIMENTAL SETUP}

The experimental arrangement is shown in Fig. 1. A $1.1 \mathrm{MHz}$ HIFU source (Sonics Concepts, Bothell, WA) was driven by a function generator and amplified. An agargraphite phantom [3] ( 1.1 MPa cavitation threshold) was positioned such that the HIFU focus was approximately $15 \mathrm{~mm}$ from the proximal edge of the phantom. Two focused $15 \mathrm{MHz}$ broadband transducers ( 650 $\mu \mathrm{m}$ focal width) were used as passive 
cavitation detectors (PCD) in order to monitor cavitation activity simultaneously in two regions. One transducer ('focal PCD') was positioned confocal with the HIFU transducer while the other transducer ('pre-focal PCD') was set in an articulated holder, such that its focus could be positioned along the HIFU transducer axis anywhere in the pre-focal region. The signals from both PCDs were amplified and high-pass filtered above $5 \mathrm{MHz}$ for the purpose of removing the HIFU fundamental and its lower harmonics. The filtered signals were processed by analog RMS-to-DC conversion chips (AD8361) and digitized via an SCXI-1120 signal conditioning module and 12-bit data acquisition card (6 ksamples/sec). Since the PCD signal is proportional to RMS pressure, it is indicative of the square root of the radiated sound power from inertial bubble activity in the sensing zone. The voltage into the HIFU transducer was measured with a high-impedance probe in order to determine the focal pressure, which was found by calibrating the transducer in water. We then accounted for the phantom characteristics using an FDTD simulation of the nonlinear pressure field coupled to the measured acoustical properties of the phantom material [6].

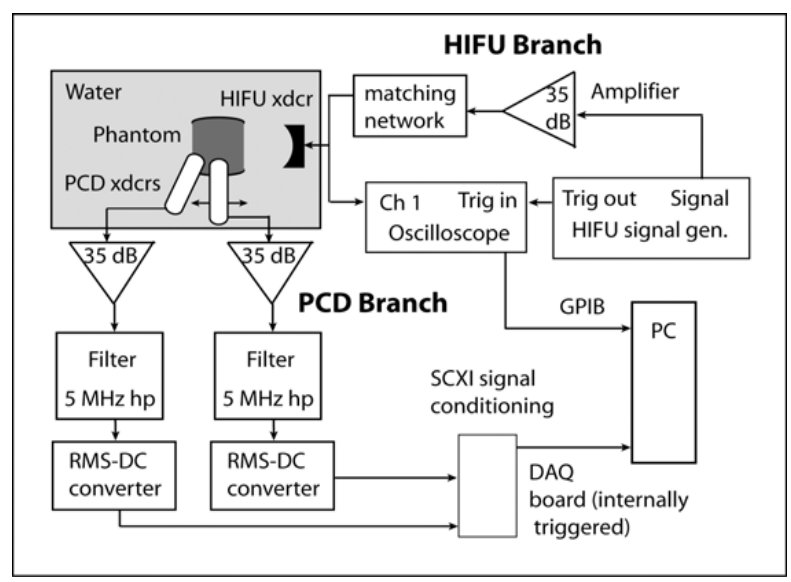

FIGURE 1. Experimental arrangement.

We monitored the PCD signal as a function of space and time for continuous-wave insonation at $3 \mathrm{MPa}$ for $6 \mathrm{~s}$. The focal PCD was fixed confocal with the HIFU source, while pre-focal cavitation activity was measured at five positions (1-5 $\mathrm{mm}$ from the focal plane) along the HIFU axis. Each pre-focal position was investigated using a fresh phantom and the suite of focal measurements proved repeatable.

\section{RESULTS AND DISCUSSION}

The cycle-averaged radiated power from the acoustic emissions from an inertial cavitation bubble was computed from the radius-vs-time curves [3]. Since we do not know our bubble sizes a priori the 'optimum' initial bubble size that radiated the maximum power at each temperature was used in generating Fig. 2, which clearly indicates a sharp decline in radiated power with increasing temperature. This change can be explained largely by the corresponding increase in vapor pressure from 
approximately $2 \mathrm{kPa}$ at $20^{\circ} \mathrm{C}$ to atmospheric pressure at $100^{\circ} \mathrm{C}$; the elevated vapor pressures serve to cushion the inertial bubble collapse. Thus, at higher temperatures, bubbles produce lower-amplitude signals and are less readily detected using passive sensing techniques. More importantly, these bubbles will play a reduced role as heat sources (assuming a relatively constant bubble population). This is a critically important point if one endeavors to use inertial cavitation to accelerate heating. As the tissue heats up, these inertial cavities become neutralized.

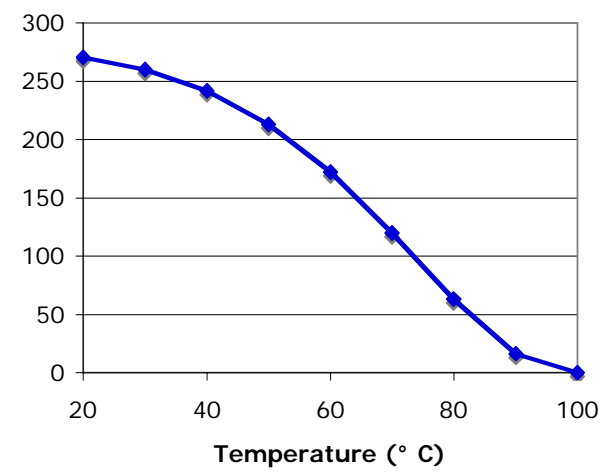

FIGURE 2. Cycle-averaged radiated power for a single bubble in water as a function of ambient temperature. The driving pressure amplitude is $3 \mathrm{MPa}$, the driving frequency is $1.1 \mathrm{MHz}$.

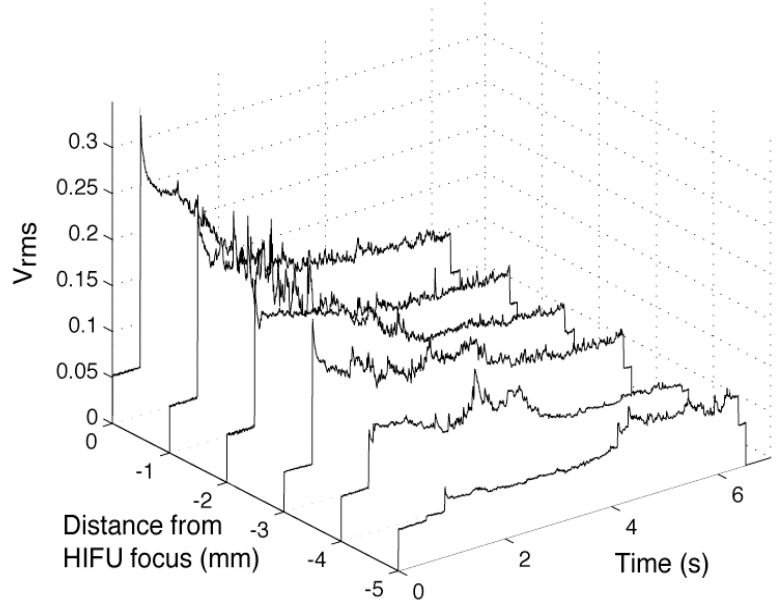

FIGURE 3. Cavitation signals over time as measured at discrete locations near the focus by the PCDs. The HIFU was on from $0.5-6.5 \mathrm{~s}$ at a $3 \mathrm{MPa}$ focal pressure.

The PCD measurements throughout the $1-5 \mathrm{~mm}$ pre-focal region are displayed in Fig. 3. The cavitation signals at and near the focus $(0,-1,-2 \mathrm{~mm})$ decrease steadily over time, with the sharpest decline occurring at the focus, where the tissue heats up fastest. The signals at -3 and $-4 \mathrm{~mm}$ decrease initially, followed by an increase in the PCD signal from about 2-3.5 s. The elevation is likely due to increasing cavitation activity brought on by a decrease in the nucleation threshold with temperature plus the contribution of additional HIFU energy backscattered from the non-inertial bubbles in 
the focus. Eventually, the tissue heats up sufficiently to neutralize inertial cavitation, and the PCD signal diminishes. Shielding from bubbles in this pre-focal zone could also contribute to the observed decay in the signal at and near the focus.

The signal at $-5 \mathrm{~mm}$ signal shows little activity until about $4 \mathrm{~s}$, where it suddenly jumps to an elevated level for the remainder of the insonation. Presumably, the advancing cavitation zone reaches the $-5 \mathrm{~mm}$ position at the $4 \mathrm{~s}$ mark. Moreover, the onset of this pre-focal bubble activity coincides with the rapid reduction in bubble emissions at -3 and -4 s; pre-focal bubbles acoustically shield the focal zone. These results indicate both bubble shielding and temperature effects are implicated in nearfocus reductions in inertial cavitation activity. We believe this behavior contributes to deleterious pre-focal heating and observations of tadpole lesion formation.

\section{SUMMARY AND CONCLUSION}

The presence of inertial cavitation has been shown to assist HIFU treatment by increasing the temperature in a region confined to the bubble activity, but the initiation of pre-focal cavitation can lead to energy deposition in undesired locations and blockage of energy to the target region. Our initial goal was to improve our understanding of how the cavitation signal changes over time, and it appears now that the signal decrease observed at the focus is due to both bubble shielding and decreased bubble emissions at elevated temperatures. A more important lesson, however, may be that at high temperatures the diminished heating contribution of inertial bubbles may render them ineffective as sources of enhanced HIFU heating.

\section{ACKNOWLEDGMENTS}

We gratefully acknowledge the financial support of the U.S. Army, Award Number DAMD17-02-2-0014 and the Center for Subsurface Sensing and Imaging Systems via NSF ERC Award Number EEC-9986821.

\section{REFERENCES}

1. Watkin, N.A., ter Haar, G.R., Rivens, I., Ultrasound Med. Biol., 22, 483-491 (1996).

2. Bailey, M.R., et al., Acoustical Phys., 49, 369-388 (2003).

3. Holt, R.G., Roy, R.A., Ultrasound Med. Biol., 27, 1399-1412 (2001).

4. Yang, X., Roy, R.A., Holt, R.G., J. Acoust. Soc. Am., 116, 3423-3431 (2004).

5. Farny, C.H., Wu, X., Murray, T.W., Holt, R.A., Roy, R.A., ARLO, 6, 138-143 (2005).

6. Thomas, C.R., Farny, C.H., Coussios, C.-C., Roy, R.A., Holt, R.G., ARLO, 6, 182-187 (2005).

7. Prosperetti, A., Crum, L.A., Commander, K.W., J. Acoust. Soc. Am., 82, 502-514 (1988).

8. Kamath, V., Prosperetti, A., J. Acoust. Soc. Am., 84, 1538-1548 (1989).

9. Hill, C.R., Physical Principles of Medical Ultrasonics, NY, Ellis Harwood Limited, 1986.

10. CRC Handbook of Chemistry and Physics, $80^{\text {th }}$ ed., Boca Raton, FL, CRC Press, 2000.

11. Prosperetti, A., Hao, Y., Phil. Trans. Royal Soc. London Ser. A, 357, 203-223 (1999).

12. Kestin, J., Sokolov, M., Wakeham, W.A., J. Phys. Chem. Ref. Data, 7, 941-948 (1978).

13. Maroto, J.A., de las Nieves, F.J., Quesada-Perez, M., Eur. J. Phys., 25, 297-301 (2004). 